\title{
Bodyflow as a Rehabilitation Strategy in the Treatment of Complex Regional Pain Syndrome
}

\author{
Tahuser Martínez Lara ${ }^{1}$, Neysa Margarita Pérez Rodríguez ${ }^{2}$, Pavel Yosvany Suárez Guevara ${ }^{\text {* }}$ \\ ${ }^{1}$ Physical Medicine and Rehabilitation of the International Orthopedic Scientific Complex "Frank País", Faculty Finlay -Albarrán, University \\ of Medical Sciences of Havana, Havana, Cuba \\ ${ }^{2}$ Physical Medicine and Rehabilitation of the University of Medical Sciences, Faculty Finlay -Albarrán, University of Medical Sciences of \\ Havana, Havana, Cuba \\ ${ }^{3}$ Comprehensive General Medicine of the Polyclinic Elpidio Berovides, Faculty Finlay -Albarrán, University of Medical Sciences of Havana, \\ Havana, Cuba
}

Email address:

pavelsg@infomed.sld.cu (P. Y. S. Guevara), neysampr@gmail.com (N. M. P. Rodríguez)

${ }^{*}$ Corresponding author

\section{To cite this article:}

Tahuser Martínez Lara, Neysa Margarita Pérez Rodríguez, Pavel Yosvany Suárez Guevara. Bodyflow as a Rehabilitation Strategy in the Treatment of Complex Regional Pain Syndrome. Pathology and Laboratory Medicine. Vol. 5, No. 1, 2021, pp. 6-9.

doi: $10.11648 /$ j.plm.20210501.12

Received: March 29, 2021; Accepted: April 22, 2021; Published: April 30, 2021

\begin{abstract}
Background: Complex regional pain syndrome is a progressive painful chronic neuropathy with autonomic nervous system dysfunction, bone demineralization, and muscle weakness; whose appearance is associated with a traumatic event such as fracture, surgery or cardiovascular event, among others, which usually affects one or more extremities. Its treatment constitutes a great challenge for science, currently settling in physiotherapy the greatest expectations for the future for rehabilitation. Bodyflow is a low frequency electro stimulator that can be applied to reduce edema, stimulate circulation and lymphatic drainage. The method seeks to contract the tunica media of the arteries and veins, as a facilitating means for the transport of lymph and blood. Stimulation is applied to the muscle, using suction cups and electrodes located in different body segments. Objective: To present a review on Bodyflow as an effective rehabilitation strategy in the treatment of complex regional pain syndrome. Methods: To achieve this purpose, electronic and library searches of national and foreign medical journals indexed in Scielo, Imbiomed and Pubmed were carried out over a 10-year horizon. Conclusions: There are few studies that suggest the use of bodyflow therapy as an alternative for the treatment of complex regional pain syndrome and this article suggests its use due to its analgesic and motor effects.
\end{abstract}

Keywords: Complex Regional Pain Syndrome, Reflex Sympathetic Dystrophy, Sudeck Syndrome, Bodyflow, Vascular Neurostimulation, Physiotherapy, Rehabilitation

\section{Introduction}

The term Reflex Sympathetic Dystrophy Syndrome, introduced by Evans in the medical literature in 1946, has received various names, all of them imprecise, some denoting the origin of the disorder, others the mechanisms of pain and others its evolution. Thus, it has been called: causalgia syndrome (major and minor), sudeck's atrophy, algodystrophy, algoneurodystrophy, reflex neurovascular dystrophy, Steinbrocker syndrome, post-traumatic osteoporosis, post-traumatic dystrophy, post-traumatic vasospasm, post-traumatic chronic edema, sympathalgia, etc. Starting in 1994, the International Association for the Study of Pain (IASP) brought together all these definitions under the term COMPLEX REGIONAL PAIN SYNDROME to denote regional pain and autonomic and sensory changes. that exceed a traumatic phenomenon in intensity, magnitude, or both. Thus, Complex Regional Pain Syndrome was classified as Type-I (Reflex Sympathetic Dystrophy) and Type-II (Causalgia). [1, 2]

TYPE I or RSD: predominant in the pediatric population, and may appear after minor trauma or fracture, but without defined nerve injury. 
TYPE II or Causalgia: (also known as causalgia), rare in children, produced by a defined nerve injury1. [3, 4]

The worldwide incidence ranges from 2 to $25 \%$. It is difficult to find epidemiological data considering the difficulty of diagnosing it. [5] it can occur at any age, but it is more frequent in women between 40-60 years of age, although it is being diagnosed more and more among adolescents and young people, without presenting ethnic preferences. [6]

What happens specifically in CRPS is that the sympathetic system (SS) of the autonomic nervous system (ANS) is abnormally activated, producing substances that activate nociceptors and perpetuate pain, while permanent vasomotor disorders occur. [7]

In two-thirds of cases, a triggering episode can be identified that may be a trauma caused by injuries, secondary to high-speed impacts by: bullets, shrapnel, or a traffic accident. Less frequently, it can be caused by fractures, orthopedic or vascular surgeries with peripheral nerve injuries, and is also associated with diseases such as osteoarthritis, intervertebral disc disease, systemic lupus erythematosus, myocardial infarction, and acute stroke, although it can also occur without apparent injury. [8,9]

There is no laboratory test to diagnose it; therefore, the clinician must evaluate and document all symptoms and signs present in order to establish the diagnosis. It is crucial to diagnose this disease in its early stage, as it can spread to all extremities, making rehabilitation much more difficult3. If the diagnosis is made early, physical therapy can be used, in order to increase mobility in the affected limb and alleviate the chronic pain and permanent deformities that it causes. [10-12]

The pain, disproportionate to the initial episode, is continuous and intensifies when trying to mobilize the affected joints, is spontaneous, allodynia, hyperalgesia and / or hyperpathy are not limited to the territory of a single peripheral nerve. Edema, cutaneous vascular flow abnormalities, and sudomotor abnormalities are clinically noticeable by distal or mesomelic lesions. It evolves in three clinical phases:

1. Phase 1: characterized by severe, burning pain and distal end swelling that appears weeks or months after the triggering process. The pain is usually continuous, but with periods of exacerbation. There may be excessive sweating and localized hypertrichosis (excessive hair growth).

2. Phase 2: In three to six months the skin gradually thins and becomes shiny and cool. The manifestations of these first two phases can overlap.

3. Phase 3: In the following three to six months, irreversible changes occur in the skin and bones, causing atrophy (decrease in volume and weight of an organ) of the skin and subcutaneous cellular tissue, producing an irreversible contracture in flexion. of the hand or foot, typical of the disease. [7]

Patients report that pain worsens over time, rather than improves, and can be accentuated by emotional stress.
Muscle spasms, osteomalacia (softening of the bones), motor problems, and changes in the nails and skin may also appear near the wound site that is hot, red, and shiny and then turns cold and bluish. [7]

Many authors affirm that physiotherapy is one of the most important procedures for the improvement of CRPS, since it helps to reduce pain more quickly, to regain mobility and to reduce the edema of the limb. [13, 14]

The current pillars of the conservative treatment of CRPS or RSD worldwide are based on three fundamental aspects: Medications, Physical and rehabilitative therapy and Surgery with the aim of recovering functions and reducing symptoms. [15]

Physiotherapy treatment is essential in this type of problem to improve symptoms, it is necessary to start it early to avoid chronification. [16] Physiotherapy plays a basic role as it has an arsenal of procedures that influence pain and circulatory ecstasy. The early application of the treatment provides greater efficacy. $[17,18]$

The main objective of the rehabilitative treatment is to achieve functional restoration. Taking into account that the treatment must be individualized and adapted to the characteristics of each patient. In the presence of a Type I CRPS, either stage I, II or III, or a Type II CRPS, treatment should be governed by the following guidelines:

1. Pain relief and edema control.

2. Increased mobility to ensure the integrity of the joint ranges, adequate gliding of the tendons and nerve mobilization.

3. Improved muscle performance.

4. Improved total body circulation.

5. Desensitization of the affected area.

6. Functional and occupational rehabilitation programs (ergonomic reconditioning). [17, 19]

For this reason, we propose in this study to present a review on Bodyflow as an effective rehabilitation strategy in the treatment of complex regional pain syndrome.

\section{Methods}

As a search strategy, the Health Information Locator (LIS) located on the Cuban Rehabilitation Medicine portal at Infomed was accessed. A systematic review was carried out, by means of electronic search and in libraries of national and foreign medical journals indexed in Scielo, Imbiomed and Pubmed for 10 years. The search strategy was developed in the period from January to February 2021. For the search, the following keywords were used: complex regional pain syndrome, reflex sympathetic dystrophy, Sudeck syndrome, bodyflow, vascular neurostimulation, physiotherapy, rehabilitation.

\section{Developing}

The Bodyflow is a low frequency electro stimulator that can be applied to reduce edema, stimulate circulation and lymphatic drainage. [20] 
It is an exclusive treatment with advanced technology that allows athletes and patients to recover more quickly from injuries, facilitating the flow of body fluids such as blood by stimulating smooth muscle, using a specific electro stimulation frequency, whether by electrotherapy, vacuum or combination depending on the patient. [21]

The method seeks to contract the tunica media of the arteries and veins, as a facilitating means for the transport of lymph and blood. Stimulation is applied to the muscle, using suction cups and electrodes located in different body segments. [22]

Low frequency currents have an "analgesic and motor effect". The Bodyflow ${ }^{\circledR}$ electrical stimulator uses a proprietary low frequency of $1.52 \mathrm{~Hz}$ and a pulse width of 6ms. [21]

The Bodyflow ${ }^{\circledR}$ currents are isolated impulses and each one gets their own job. Finally, the team uses an exponential waveform, with progressive rise and fall, this type of wave has a lower capacity for membrane depolarization. Regarding the dosage of intensity, two conditions are taken into account, if you want to have a sensitive response, the scale is indicated by the subjectivity of the user; If the goal is to have a motor response, it will depend on palpation and consideration of the level of muscle contraction achieved. In the case of Bodyflow ${ }^{\circledR}$, the intensity is set taking into account the two conditions mentioned above. [21]

Vascular neurostimulation uses suction cup electrons which are placed on the nodes at a rate of 6 to 8 beats per minute and adhesive electrodes on the striated muscles in order to achieve an efficient nodal pump and obtain complete lymphatic emptying. Since the lymph nodes do not contain the smooth muscles, the suction cup electros perform a negative pressure that allows to mobilize the fluids contained in the lymph nodes and prevent lymph node congestion from occurring. [20]

The Bodyflow frequency has been set to specifically stimulate smooth muscle and has been shown to increase blood and lymphatic flow. People with vascular diseases often have symptoms related to insufficient blood flow, especially in the lower legs and feet. Certain conditions, such as Diabetes-associated Peripheral Vascular Disease, elderly care, and conditions associated with symptoms such as cold feet, leg pain, restless legs and foot and leg ulcers, peripheral neuropathy (nerve damage in the extremities) and pain associated with poor circulation can greatly benefit from Bodyflow ${ }^{\circledR}$ treatment. [20]

Contraindications:

1. Pregnant.

2. Active malignant diseases.

3. Major inflammatory processes.

4. Fever.

5. Venous thrombosis.

6. Pacemakers and with heart problems. [20]

According to Porro et al, [23] physiotherapy plays a fundamental role, having a group of procedures that influence pain and circulatory stasis to subsequently improve mobility, trophism and stiffness, but there is no standard treatment.

Many authors suggest that treatment be with a multidisciplinary, individualized, early and intensive approach, where a combination of pharmacological, physical, occupational and psychological therapies is used, with a view to desensitizing pain, minimizing edema, reducing atrophy, as well as normalizing sensitivity and movement patterns using neuromuscular reeducation techniques to develop strength and range of motion and increase functionality, to avoid chronicity and irreversible sequelae of CRPS. [24, 25]

\section{Conclusions}

The treatment of Sudeck is a treatment to improve symptoms. For this, a multidisciplinary treatment with medications, physical therapy and psychological therapy must be considered. Physiotherapy treatment is essential in this type of problem to improve symptoms, it is necessary to start it early to avoid chronification. It is necessary to highlight the individuality of the user, which makes the evaluation, diagnosis and treatment, are specific and that there are no unique protocols to apply, because the actions they vary according to the reception that the user makes of each one.

There are few studies that suggest the use of bodyflow therapy as an alternative for the treatment of complex regional pain syndrome and this article suggests its use due to its analgesic and motor effect.

\section{Conflict of Interests}

The authors declare that they have no conflict of interest.

\section{Authors' Contribution}

Tahuser Martínez Lara: Conceptualization, research, methodology

Neysa Margarita Pérez Rodríguez: Writing of the original draft, writing

Pavel Suárez Guevara: Revision, supervision, editing

\section{References}

[1] Rehabilitation approach to complex regional pain syndrome type I. 2019 [accessed: 01/11/2021]. Available at: www.revreumatologia.sld.cu/index.php/reumatologia/article/v iew/15/369

[2] Buller Viqueira, E. et al. Reflex sympathetic dystrophy: genetic predisposition? 2016. rev clin med fam 2016 [accessed 02/23/2021]; 9 (3): 237-242 Available at: scielo.isciii.es $>$ scielo $>$ pid $=$ S1699-695X20160003000..

[3] Del Valle González Germán MF, Vega Hernández P, DíazDelgado Peñas R. Complex regional pain syndrome. Diagnostic protocol pediatr. 2020 [accessed 02/23/2021]; 2: 325-334. 
[4] Alvizo-Pérez ME, Gallardo-Martínez ER, Rodríguez-Aceves G. Reflex Sympathetic Dystrophy Type I, Sudeck or Algodystrophy: Report of a case. Medical Journal MD; 2017 [accessed: 01/11/2021]; 8 (3).

[5] Sanz Arrabal I. Complex regional pain syndrome (CRPS) or Südeck's disease. Reduca (Nursing, Physiotherapy and Podiatry) Series Podiatric clinical sessions. 2016 [accessed 03/16/2021]; 8 (3): 1-7. Available at: revistareduca.es >index.php> issue $>$ view〉 showToc

[6] Hernández Zayas ET AL. Clinical, radiographic and rehabilitative aspects in patients with complex regional pain syndrome. 2018 [accessed 03/16/2021]; 22 (2). Available at: www.medisan.sld.cu >index.php> san >article> view

[7] Larrarte Martínez J, Almeida Sosa M, Cabreja López G, Reyes Pineda Y, Morales Cepero R et al. Reflex Sympathetic Dystrophy, etiological factors and evolution. Cuban Journal of Rheumatology 2000 [accessed: 01/11/2021]; Volume II, Num 1.

[8] Südeck Syndrome, what is it and how is it treated? 2020 [accessed: 01/11/2021] Available at: www.clinicadanireig.com $>$ sudeck-syndrome-that-is...

[9] Weissmann R, Uziel Y. Pediatric complex regional pain syndrome: a review. Pediatr. Rheumatol. Online J. 2016 [accessed 02/23/2021]; 2:29 PM Available at: pubmed.ncbi.nlm.nih.gov >

[10] 1Abu-Arafeh H, Abu-Arafeh I. Complex regional pain syndrome in children: a systematic review of clinical features and movement disorders. Pain Manag. 2017 [accessed 02/23/2021]; 7: 133 .

[11] Dickson SK. Including Parents in the Treatment of Pediatric Complex Regional Pain Syndrome. Pediatr Nurs. 2017 [accessed 02/23/2021]; 43: 16-21.

[12] Giovanni Cucchiaro, Kevin Craig, Kerri Marks, Kristin Cooley, Thalitha Kay et al. Short- and long-term results of an inpatient program to manage Complex Regional Pain Syndrome in children and adolescents. Br J Pain. 2017 [accessed 02/23/2021]; 11 (2): 87-96.

[13] Gisela Pendón, Adrian Salas, Mercedes García, Dora Pereira. Complex Regional Pain Syndrome Type 1: Analysis of 108 Patients. Elsevier. 2017. [accessed 01/06/2021]Available at: www.reumatologiaclinica.org> en-estadisticas-S217357.

[14] Hernández Porras BC, Plancarte Sánchez R, Alarcón Barrios S, Sámano García M.. Complex regional pain syndrome: review. Surgery and Surgeons. 2016 [accessed: 01/06/2021]; 85 (4): 366-374.

[15] Smart KM, Wand BM, O'Connell NE. Physiotherapy for pain and disability in adults with complex regional pain syndrome (CRPS) types I and II. Surgery and Surgeons 2017. 85 (4): 366-374.

[16] Physiotherapy treatment of reflex sympathetic dystrophy or Südeck at FisioClinics Logroño. 2019 [access11 / 01/2021] Available at: https://logrono.fisio-clinics.com/distrofiasimpatico-refleja-o-sudeck

[17] Esparza-Ramos Susana Berenice, Guzmán- Esquivel Javier, Herrera-Gamboa Luis Ovidio. Complex regional pain syndrome. Rev. Luxmedica. 2016. [accessed: 01/06/2021] Available at: revistas.uaa.mx , luxmedica > article > download

[18] Smart K, Wand B, O'Connell N. Physiotherapy for pain and disability in adults with complex regional pain syndrome (CRPS) types I and II. 2016 [accessed: 01/06/2021] Available at: $10.1002 / 14651858 . C D 010853$

[19] A. Gómez-García de Paso, A. Muñoz-Martín, F. J. Robaina. Medullary neuroelectrostimulation in Type I complex regional pain syndrome. Case study. Rev. Soc. Esp. 2007 [accessed: 01/06/2021]; vol. 14 no. 8 .

[20] BODY FLOW | Clínica Internacional Siboney - Institutions. [accessed: 01/06/2021] Available at: https://instituciones.sld.cu/cis/aparatologia/calidad-devidad/body-flow/

[21] Ospina Castro A L. Effect of vascular neurostimulation on smooth muscle in post-exercise recovery in athletes. [Master's Thesis], National University of Colombia-Bogotá. 2015

[22] Sostaric S, Pearce A, Gatt B, McKenna M, Stathis C, Goodman C. Effects of mild electro-stimulation (Bodyflow $\mathrm{TM}$ ) treatment on healthy humans following exercise induced muscle damage. Center for aging, Rehabilitation, Exercise \& Sport. Victoria University, Melbourne, Australia. 2007

[23] Porro Novo J, Estévez Perera A, Prada Hernández DM, Garrido Suárez B, Rodríguez García A. Rehabilitative approach to complex regional pain syndrome type I. Rev Cubana Reumatol. 2012 [accessed 02/23/2021]; 14 (20). Available at:

http://www.revreumatologia.sld.cu/index.php/reumatologia/art icle/view/15

[24] Harald Breivik. Invesive Intervention for "Intractable" Complex Regional Pain Syndromes (CRPS)? Elsevier. 2017 [accessed: 01/06/2021] Available at: ous-research.no , home , pain> $G$.

[25] Figueroa Huaylla, Noemí Rebeca. Physiotherapeutic treatment in complex regional pain syndrome. Lima Peru. 2017[accessed: 01/06/2021] Available at: core.ac.uk , download $p d f$. 